\title{
Attention Alters Perceived Attractiveness
}

\section{Citation}

Störmer, Viola S, and George A Alvarez. "Attention Alters Perceived Attractiveness."

Psychological Science 27, no. 4 (2016): 563-71.

\section{Permanent link}

http://nrs.harvard.edu/urn-3:HUL.InstRepos:41302421

\section{Terms of Use}

This article was downloaded from Harvard University's DASH repository, WARNING: This file should NOT have been available for downloading from Harvard University's DASH repository.

\section{Share Your Story}

The Harvard community has made this article openly available.

Please share how this access benefits you. Submit a story.

Accessibility 


\title{
Attention Alters Perceived Attractiveness
}

\author{
Viola S. Störmer and George A. Alvarez \\ Department of Psychology, Harvard University
}

Psychological Science

2016, Vol. 27(4) 563-571

(C) The Author(s) 2016

Reprints and permissions:

sagepub.com/journalsPermissions.nav

DOI: $10.1177 / 0956797616630964$

pss.sagepub.com

\begin{abstract}
Can attention alter the impression of a face? Previous studies showed that attention modulates the appearance of lower-level visual features. For instance, attention can make a simple stimulus appear to have higher contrast than it actually does. We tested whether attention can also alter the perception of a higher-order property-namely, facial attractiveness. We asked participants to judge the relative attractiveness of two faces after summoning their attention to one of the faces using a briefly presented visual cue. Across trials, participants judged the attended face to be more attractive than the same face when it was unattended. This effect was not due to decision or response biases, but rather was due to changes in perceptual processing of the faces. These results show that attention alters perceived facial attractiveness, and broadly demonstrate that attention can influence higher-level perception and may affect people's initial impressions of one another.
\end{abstract}

\section{Keywords}

attention, perception, face perception, visual attention

Received 6/7/15; Revision accepted 1/13/16

It is well established that attention improves behavioral performance in a variety of tasks. For instance, directing attention to a particular location results in better and faster detection and discrimination of stimuli appearing at that location (LaBerge, 1995; Pashler, 1998). Although these behavioral benefits of attention are well known, it is a separate question whether attention alters phenomenology, that is, how an object looks to the observer. This question has been a matter of debate since the earliest days of psychology. James (1890) and Helmholtz (1866) hypothesized that attention intensifies sensory experience, whereas Fechner (1882) asserted that attention does not bias people's impressions.

Previous research has shown consistent effects of attention on the appearance of simple stimulus features. In a series of psychophysical experiments, Carrasco and her colleagues found that attention increased perceived stimulus contrast (Carrasco, Fuller, \& Ling, 2008; Carrasco, Ling, \& Read, 2004; Liu, Abrams, \& Carrasco, 2009; see also Störmer, McDonald, \& Hillyard, 2009), perceived spatial frequency (Gobell \& Carrasco, 2005), perceived color saturation (Fuller \& Carrasco, 2006), perceived motion coherence (Liu, Fuller, \& Carrasco, 2006), perceived speed (Anton-Erxleben, Herrmann \& Carrasco, 2013), and perceived flicker rate (Montagna \& Carrasco,
2006). All of these studies used simple visual stimuli, such as sinusoidal gratings (i.e., Gabor patches), line shapes (i.e., Landoldt rings), or dots. These stimuli are highly valuable for studying low-level mechanisms of sensory processing and make it possible to investigate effects of attention on perception of properties that are objectively measurable in the stimulus (e.g., contrast, color saturation). Although observers can judge these same objective properties in complex, real-world stimuli, such as faces, they can also make subjective judgments that cannot be measured objectively, such as judgments of how attractive a face is. To date, it remains unknown whether attention modifies the subjective experience of real-world stimuli.

In the present study, we investigated whether exogenous attention alters high-level perception of real-world stimuli, namely, faces. Of all the stimuli that people encounter in the real world, they interact most with faces, from which a wide range of socially relevant properties can be extracted. Moreover, there is considerable overlap

\section{Corresponding Author:}

Viola S. Störmer, Harvard University, Department of Psychology, 33

Kirkland St., Cambridge, MA 02138

E-mail: vstormer@fas.harvard.edu 
in different individuals' impressions of faces. People judge personality traits on the basis of faces (Efran, 1974; Todorov, Mandisodza, Goren, \& Hall, 2005), and they can do so after only brief exposure to a face image ( $100 \mathrm{~ms}$; Willis \& Todorov, 2006). Thus, impressions of faces are formed quickly, and they have powerful consequences for subsequent interactions.

We examined whether attention influences subjective judgments of facial attractiveness. Facial attractiveness has been associated with facial symmetry (Perrett et al., 1999; Rhodes, Proffitt, Grady, \& Sumich, 1998) and facial averageness (Langlois \& Roggman, 1990; Rhodes \& Tremewan, 1996), as well as with facial hormone markers, such as larger lips in the case of female faces and more lateralized cheekbones in the case of male faces (Cunningham, Barbee, \& Pike, 1990; Perrett et al., 1998; for a review, see Rhodes, 2006). Furthermore, recent studies showed that female faces with higher contrast around the eye region (eyebrows, eyelashes) and the mouth are judged to be more attractive (Russell, 2003, 2009). However, these studies mostly examined features intrinsic to faces and did not assess the extent to which facial attractiveness depends on the observer's focus of attention. Finding that attention influences perceived facial attractiveness would place significant constraints on accounts of face perception in general.

To test whether attention alters perceived attractiveness of faces, we used a modified version of the attentional-cuing paradigm initially developed by Carrasco et al. (2004). This paradigm measures the effects of exogenous attention on perceptual processing while carefully controlling for decision or response biases. In each trial of our version of the task, two gray-scale face images were briefly presented at the same time on the left and right sides of a computer screen; one was shifted upward, and the other one was shifted downward along the vertical axis. Participants were instructed to report the vertical shift (upward or downward) of the face they perceived as more attractive without moving their eyes from the center of the screen (Fig. 1a). The faces were matched in overall brightness, but the local contrast around the eye region (eyes and eyebrows) was systematically manipulated for each of the faces (Fig. 1b). To summon attention to one of the faces, we presented a task-irrelevant black dot (the attentional cue) at the left or right location immediately before the presentation of the face images. Given previous studies showing a link between female attractiveness and local contrast around the eye region (Russell, 2003, 2009), we expected participants to judge the faces with higher contrast around the eyes to be more attractive than the same faces with lower contrast around the eyes. Of principal interest was whether the exogenous cue would also influence participants' attractiveness judgments. If it did, participants would choose the face at

\section{a}

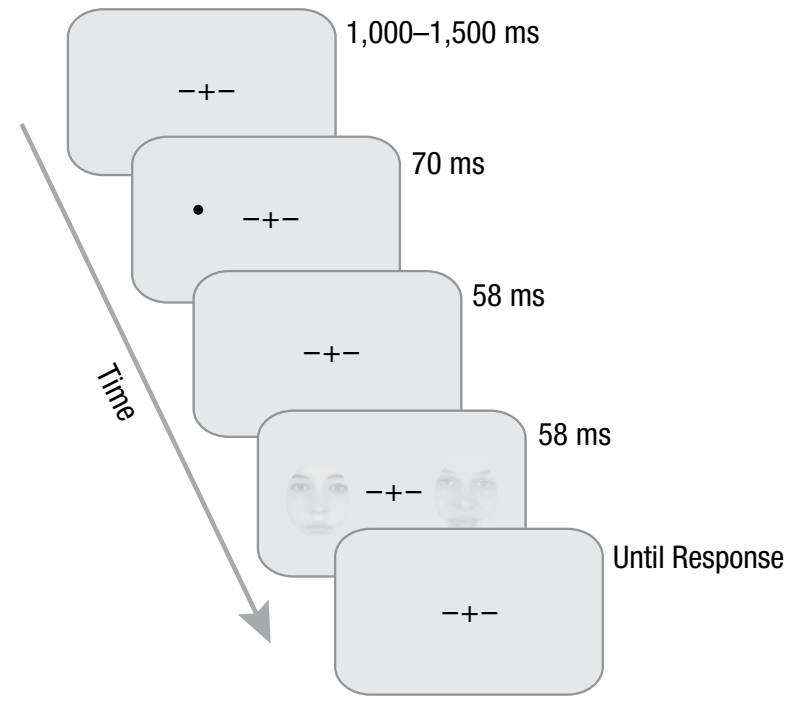

b

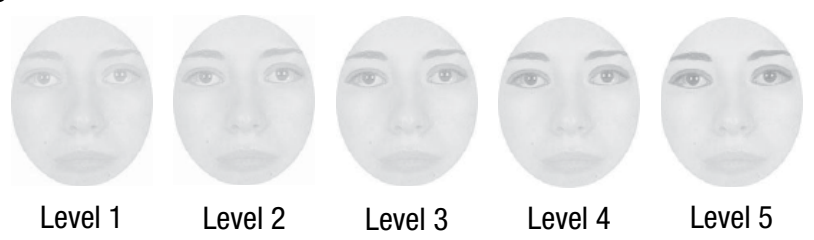

Fig. 1. Illustration of the trial sequence and examples of the stimuli. On each trial (a), two faces were presented simultaneously to the left and right of fixation; one face was shifted slightly upward, and the other face was shifted slightly downward along the vertical axis. Before the target faces were presented, a small black dot was briefly flashed at the left or right location. Each face stimulus varied across trials in its local contrast around the eye region (eyes and eyebrows). The lowest level of contrast was Level 1, and the highest level was Level 5 (b). Overall brightness was matched across all contrast levels and all face images.

the cued location consistently more (or less) often than the same face presented at the uncued location.

To preview our results, we found that participants reported the faces at the cued location as more attractive than the faces at the uncued location. These cuing effects followed a time course similar to that observed in previous exogenous-cuing studies (e.g., Carrasco et al., 2004; Nakayama \& Mackeben, 1989), which suggests that the effects we observed were due to the involuntary orienting of attention. Finally, we found that attentional cuing modulated the apparent local contrast of the faces, which suggests that the attention-induced changes in perceived attractiveness were mediated by changes in perceived contrast. Combined, these results show that attention has consequences for the perception of real-world stimuli, including the perception of individuals' attractiveness. 


\section{Experiment 1}

\section{Metbod}

Participants. Seventeen observers participated in Experiment 1. One participant did not complete the experiment because of calibration problems with the eye tracker; that person's data were thus excluded from the final analysis. The remaining 16 participants ( 8 female, 8 male) had normal or corrected-to-normal vision, were between the ages of 18 and 28 years, and gave written informed consent prior to the experiment. The number of participants was based on previous studies investigating how exogenous cuing of attention affects apparent stimulus contrast (Carrasco et al., 2004; Störmer et al., 2009). All experimental procedures were approved by the Committee on the Use of Human Subjects in Research under the Institutional Review Board for the Faculty of Arts and Sciences of Harvard University.

Stimuli. A small black fixation cross $\left(0.5^{\circ} \times 0.5^{\circ}\right)$ was presented in the center of the screen throughout the experiment. Two small horizontal lines $\left(\sim 0.5^{\circ}\right.$ long) were presented to the left and right of fixation and served as landmarks for the horizontal midline of the screen. The target display consisted of two faces (each $8^{\circ} \times 6^{\circ}$ ) that were presented to the left and right of fixation at an eccentricity of $6^{\circ}$. The face images were chosen from 20 images of female Caucasian faces (approximate age range from 20 to 30 years) taken from Bronstad and Russell's (2007) database. They were converted to gray scale and cropped such that only their inner features (no hair or neck) were visible. All the faces were matched in overall brightness $\left(104 \mathrm{~cd} / \mathrm{m}^{2}\right)$, but the contrast of the eye region was systematically manipulated for each face. Specifically, a mask (handdefined in Adobe Photoshop) covering the eyes and the eyebrows was created for each face, and the contrast within that mask was manipulated by parametrically changing the standard deviation of that section of the image using MATLAB (The MathWorks, Natick, MA). This decreased or increased the luminance differences around the eye region in the face. For each face, five different contrast levels were created. These levels were measured in terms of root-mean-square error (RMSE) of the pixels' luminance values within the masked region. Contrast levels of $0.30,0.35,0.39,0.44$, and 0.50 RMSE were used (see Fig. 1b for examples of the stimuli).

On every trial, the contrast around the eyes of one face, the standard face, was fixed at Level 3 (0.39 RMSE), and the contrast around the eyes of the other face, the test face, was randomly chosen from the five contrast levels. The left-right positions of the standard and test faces were randomized across trials. In each target display, one of the faces was shifted slightly upward from the horizontal midline, and the other face was shifted slightly downward from the midline (shift of $\sim 0.5^{\circ}$ each direction). A small black circle (diameter of $0.6^{\circ}$ ) presented on the left or right served as the visual cue; its position $6^{\circ}$ to the left or right of fixation and $1.2^{\circ}$ above fixation placed it approximately at the location in between the eyes of the subsequent face image.

Procedure. The experiment was conducted in a dimly lit room, and the stimuli were presented on a 15 -in. CRT display $(1,280 \times 1,024$ pixels; $85 \mathrm{~Hz})$ whose background color was set to gray $\left(111 \mathrm{~cd} / \mathrm{m}^{2}\right)$. Participants viewed the stimuli at a distance of $57 \mathrm{~cm}$, and a chin rest was used to stabilize their heads. Participants' gaze was tracked with an eye tracker (EyeLink 1000, SR Research Ltd., Mississauga, Ontario, Canada) to ensure fixation. The experiment was run in MATLAB using the Psychophysics Toolbox (Brainard, 1997; Pelli, 1997).

Participants were instructed to maintain their gaze on the fixation cross in the center of the gray screen throughout each experimental block. When they moved their gaze more than $1.5^{\circ}$ away from fixation, the trial was aborted. At the beginning of each trial, a black circle appeared briefly $(\sim 70 \mathrm{~ms})$ on either the left or the right side of the screen. After another $58 \mathrm{~ms}$, face images were presented simultaneously on the left and right for $58 \mathrm{~ms}$ (Fig. 1a). Thus, the stimulus onset asynchrony (SOA) between the attentional cue and the faces was $128 \mathrm{~ms}$. After the offset of the faces, the gray screen with the fixation cross was presented until the participant responded. The intertrial interval varied from 1.0 to $1.5 \mathrm{~s}$. On two thirds of the trials, two different faces were randomly selected from the set of 20 faces to be presented as the target display. One of the faces was presented at the standard contrast (Level 3), and the other face was presented at one of the test contrasts (Levels 1-5). On the other third of the trials, the exact same face was presented on the left and right at the standard contrast. These trials were included so that we could compare responses to cued and uncued faces while all physical attributes of the two faces were matched. The analyses of the effects of the attentional cue on attractiveness judgments focused on these matched-face trials.

Participants were instructed to judge the relative vertical positioning of the face that appeared more attractive to them and to respond by pressing the up- or downarrow key on a keyboard. Note that the response dimension was orthogonal to the cue dimension: Participants responded along a vertical dimension (up or down) to report the positioning of the face they perceived as more attractive, whereas the location of the cue varied along a horizontal dimension (the left or right side of space). This procedure was used to eliminate simple response biases 
(e.g., a bias to always press the button that corresponded to the location of the cue). In addition, the instructions emphasized that there were no correct or incorrect responses, and that there was no time limit for response. Participants were told that the black dot appearing prior to the onset of the target faces was task irrelevant.

The experiment consisted of 20 blocks of 60 trials each. In total, there were 80 different-face trials for each combination of test contrast (Levels 1-5) and cue condition (test face cued vs. standard face cued), and 200 matched-face trials for each cue condition. Participants completed 40 practice trials prior to the experiment to get accustomed to how briefly the faces were presented.

\section{Results}

Figure 2a shows the probability, across all trials, of the test face being chosen as more attractive than the standard face, plotted as a function of the test face's contrast. The results are shown separately for trials in which the test face was cued and trials in which the standard face was cued. There was a main effect of contrast level on the probability of the test face being chosen, $F(4,15)=$ $12.67, p=.0001, \eta^{2}=.27$. Overall, participants judged faces with higher contrast around the eye region to be more attractive than faces with lower contrast. To test the effect of the cue on attractiveness judgments, we compared responses from the matched-face trials in the two cue conditions. This allowed us to specifically examine the effect of attention on attractiveness judgments, controlling for any physical differences between the faces. Participants tended to choose the face at the cued location as more attractive than the same face at the uncued location (Fig. $2 b ; 51.9 \%$ vs. $43.2 \%$ ), $t(15)=3.21, p=.006$, $\eta^{2}=.41$. Thus, the attentional cue influenced participants' perception of attractiveness.

\section{Experiment 2}

Exogenous attention is known to affect perceptual processing for only a short time after the presentation of the attentional cue (e.g., Jonides, 1980; Nakayama \& Mackeben, 1989; Wright \& Ward, 2008). Thus, if the shift in attractiveness judgments observed in Experiment 1 was driven by exogenous attention, we would expect the cue to influence these judgments only at short cue-target SOAs. To test this, we varied the delay between the cue and the target faces in our second experiment.
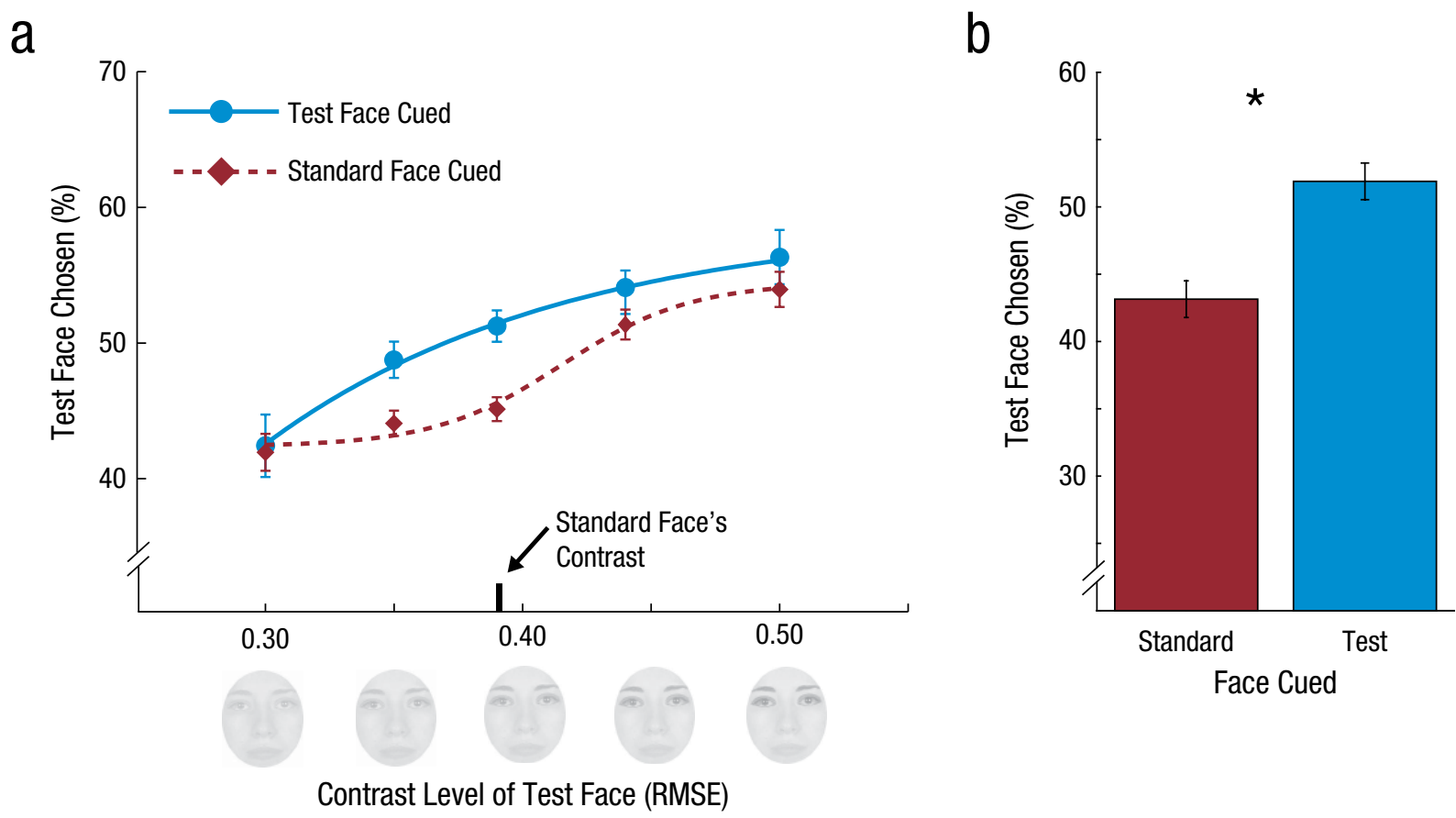

Fig. 2. Results from Experiment 1 . The psychometric functions (a) depict the percentage of all trials in which participants chose the test face as being more attractive than the standard face as a function of the test face's contrast. Results are shown separately for trials in which the test face was cued and trials in which the standard face was cued, and the data points for each cue condition have been fit using a Weibull function. The bar graph (b) shows the percentage of matched-face trials (Contrast Level 3) in which the test face was chosen as more attractive than the standard face in each of the cue conditions. Error bars represent \pm 1 SEM (within subjects). The asterisk indicates a significant difference between conditions $(* p<.05)$. RMSE $=$ root-mean-square error of the luminance values within the masked region of the face. 


\section{Metbod}

Participants. Sixteen participants completed Experiment 2. All participants (14 female, 2 male) had normal or corrected-to-normal vision, were between the ages of 18 and 28 years, and gave written informed consent prior to the experiment.

Stimuli. Experiment 2 used the same stimuli as Experiment 1 , except that only three of the contrast levels $(0.30$, 0.39 , and 0.50 RMSE) were included.

Procedure. The procedure was similar to that in Experiment 1, except that the time between the onsets of the cue and the target display (SOA) was varied (for a similar procedure, see Carrasco et al., 2004; Fuller, Rodriguez, \& Carrasco, 2008; Hsieh, Caplovitz, \& Tse, 2005; Ling \& Carrasco, 2007; Liu et al., 2006; Turatto, Vescovi, \& Valsecchi, 2007). On half of the trials, the SOA was 128 ms (short SOA), just as in Experiment 1, and on the other half of the trials, the SOA was $528 \mathrm{~ms}$ (long SOA). Trials with short and long SOAs were randomly intermixed across the experiment. Experiment 2 consisted of 16 blocks with 72 trials each. In total, there were 64 different-face trials for each combination of contrast level
(1-3), SOA (short vs. long), and cue condition (test face cued vs. standard face cued), and 96 matched-face trials for each combination of SOA and cue condition. Participants completed 48 practice trials.

\section{Results}

As in Experiment 1, we first looked for a main effect of contrast level on attractiveness judgments across all trials. Experiment 2 replicated this main effect, $F(2,15)=$ $128.27, p=.008, \eta^{2}=.12$; participants judged the highercontrast faces to be more attractive than the lowercontrast faces. Figure 3 shows the probability, across all trials, of the test face being chosen as more attractive than the standard face in each cue condition, for the short-SOA condition (Fig. 3a) and the long-SOA condition (Fig. 3b). To examine the effect of the cue in the two SOA conditions, we focused on the matched-face trials (physically identical faces at Contrast Level 3). There was no main effect of cue condition $(p=.28)$, but cue condition interacted with SOA, $F(1,15)=2.9, p=.04, \eta^{2}=.04$. Planned paired-samples $t$ tests revealed that the cue affected face attractiveness judgments on short-SOA trials $(53.1 \%$ vs. $46.9 \%), t(15)=2.31, p=.03, \eta^{2}=.26$, but not a

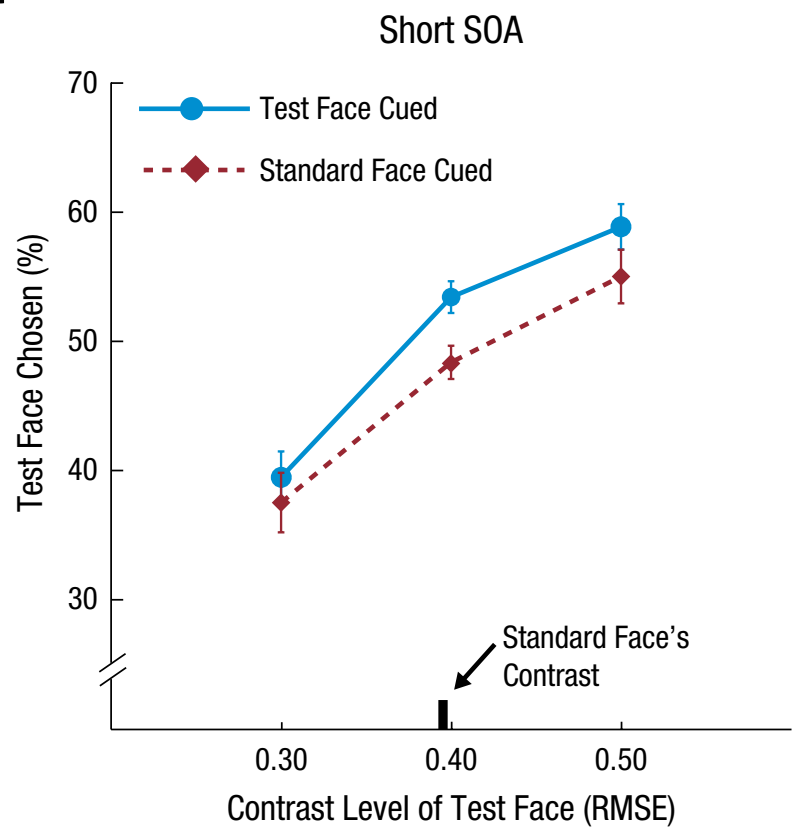

b

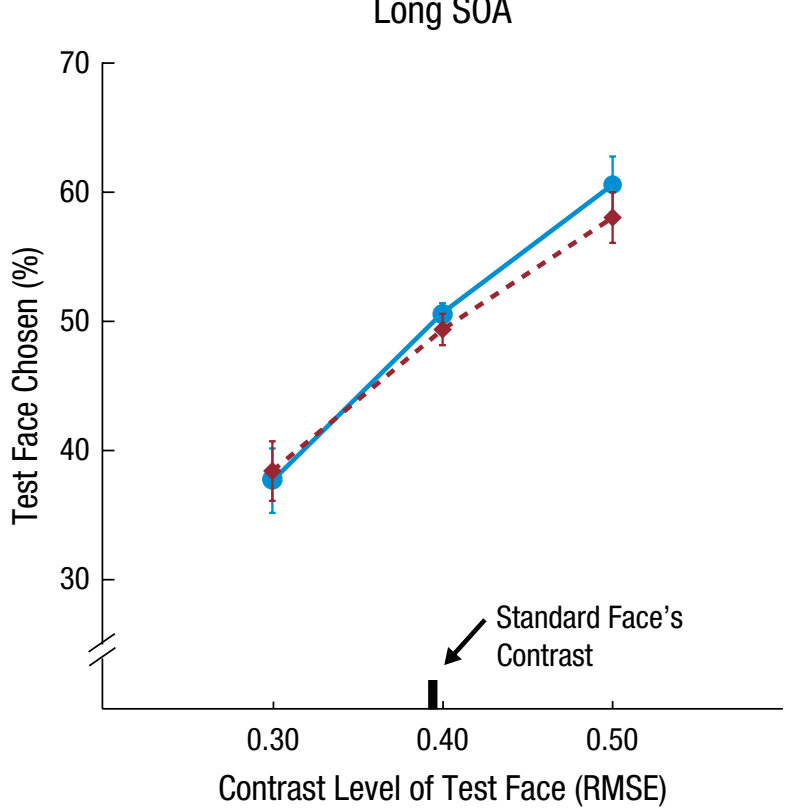

Fig. 3. Results from Experiment 2. The percentage of all trials in which participants chose the test face as being more attractive than the standard face is plotted as a function of the test face's contrast. Results for trials in which the test face was cued and trials in which the standard face was cued are shown for the two stimulus-onset-asynchrony (SOA) conditions: (a) short SOA (128 ms) and (b) long SOA $(528 \mathrm{~ms}$ ). Error bars represent \pm 1 SEM (within subjects). RMSE $=$ root-mean-square error of the luminance values within the masked region of the face. 
on long-SOA trials $(50.7 \%$ vs. $49.3 \%), p=.17, \eta^{2}=.12$. These results indicate that the shift in attractiveness judgments was due to changes in early, perceptual processing of the faces as a result of the involuntary orienting of attention to the location of the cue.

\section{Experiment 3}

In our first two experiments, participants judged faces with higher contrast around the eyes as more attractive than faces with lower contrast around the eyes, as has been found previously (Russell, 2003, 2009). Thus, we hypothesized that the attentional cue influenced attractiveness judgments by increasing apparent local contrast around the eye region of the faces. To test this hypothesis, in Experiment 3 we examined whether the attentional cue alters the apparent contrast of faces.

\section{Metbod}

Participants. Eighteen observers participated in Experiment 3. Two participants did not complete the experiment because of calibration problems with the eye tracker, and their data were excluded from the final analysis. The remaining 16 participants ( 11 female, 5 male) all had normal or corrected-to-normal vision, were between the ages of 18 and 28 years old, and gave written informed consent prior to the experiment.

Stimuli. Experiment 3 used the same stimuli as Experiment 1.

Procedure. Experiment 3 followed the same procedure as Experiment 1 except for the task instructions. Participants were asked to report the vertical positioning (upward or downward) of the face that appeared to have higher contrast around the eye region by pressing the up- or down-arrow key on a keyboard. Prior to the experiment, participants were shown three example stimuli and were told that contrast varied around the eye region. As in the other experiments, participants were told that the black dot (the attentional cue) was task irrelevant.

\section{Results}

Figure 4a shows the probability, across all trials, of the test face being chosen as having higher contrast than the standard face, as a function of the test face's contrast. Results are shown separately for trials in which the test face was cued and trials in which the standard face was cued. There was a main effect of actual contrast level on contrast judgments, $F(4,15)=27.93, p=.0001, \eta^{2}=$ 31. Participants chose the face with higher contrast around the eye region more often than the face with lower contrast around the eye region. As in the previous experiments, our main analysis focused on the matchedface trials, in which identical faces were presented at Contrast Level 3. As shown in Figure 4b, when the two faces were physically identical, participants tended to judge the face at the cued location to have higher contrast than the face at the uncued location $(55.8 \%$ vs. $45.8 \%), t(15)=2.51, p=.02, \eta^{2}=.30$. Therefore, attentional cuing increased the apparent contrast around the eye region of the face images.

On the basis of these results, we propose that an exogenous cue can change perceived facial attractiveness by enhancing the local contrast around the eye region. We ran two control experiments to rule out the alternative possibility that participants were biased to always respond to the location of the cue, regardless of what the task was. The concern that participants simply respond on the basis of the cue's location, so that differences in response patterns do not arise from differences in perceptual processing, has previously been raised with regard to the observed effects of attention on perception of simple stimuli (Prinzmetal, Long, \& Leonhardt, 2008-but see Carrasco et al., 2008; Schneider, 2006-but see Ling \& Carrasco, 2007; Schneider \& Komlos, 2008-but see Anton-Erxleben, Abrams, \& Carrasco, 2010).

To examine whether cue biases could account for our results, in our first two control experiments we followed a procedure similar to that of Experiment 1 but asked participants to report the vertical position of the face that was less attractive (rather than more attractive; see Experiment S1 in the Supplemental Material available online) or the face that had lower contrast (rather than higher contrast; see Experiment S2 in the Supplemental Material). Inverting the task instructions has previously been shown to be an effective way to test whether changes in perception judgments associated with the cue are driven by changes in perception, rather than cue biases (AntonErxleben et al., 2010; Anton-Erxleben, Henrich, \& Treue, 2007; Carrasco et al., 2004; Fuller \& Carrasco, 2006; Gobell \& Carrasco, 2005; Ling \& Carrasco, 2007; Liu et al., 2009; Montagna \& Carrasco, 2006; Turatto et al., 2007). If the cuing effects that we observed in our main experiments were due to a simple cue bias (i.e., a bias to always respond to the attended stimulus), then participants in our control experiments would choose the face at the cued location more often than the face at the uncued location despite the change in the task instructions. The results of these two control experiments did not follow this pattern, indicating that participants did not simply pick the stimulus at the location of the cue, regardless of the task instructions.

Furthermore, to ensure that the cuing effects on attractiveness judgments were not due to changes in perceived overall brightness of the faces (instead of changes in local contrast around the eyes), we ran another control 
a

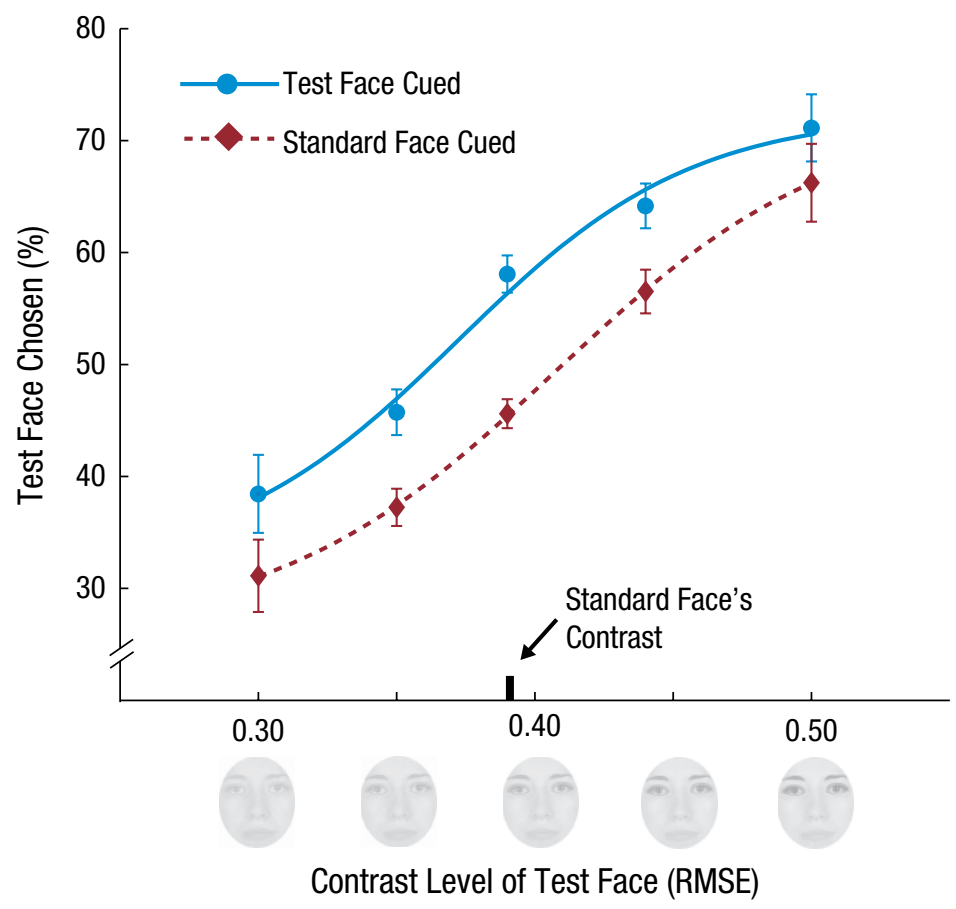

b

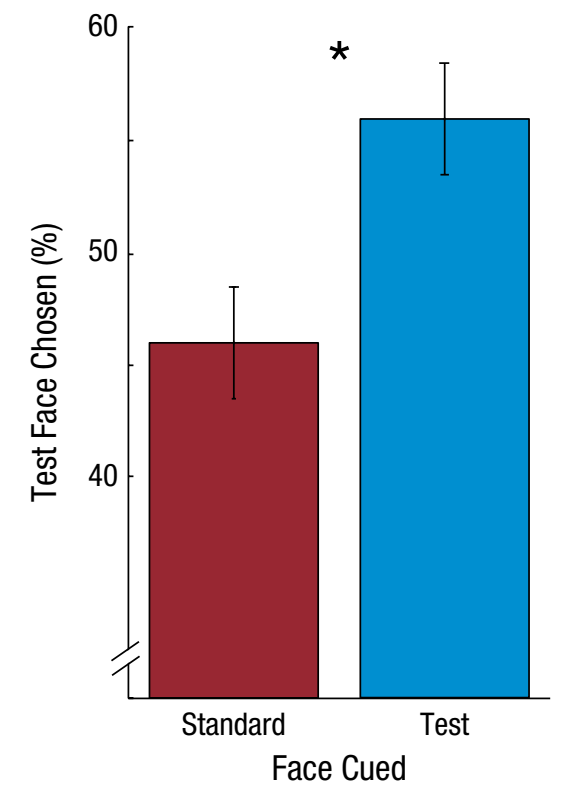

Fig. 4. Results from Experiment 3. The psychometric functions (a) show the percentage of all trials in which participants chose the test face as having higher contrast than the standard face as a function of the test face's contrast. Results are shown separately for trials in which the test face was cued and trials in which the standard face was cued. The bar graph (b) shows the percentage of matched-face trials (Contrast Level 3) in which the test face was judged to have higher contrast than the standard face in each of the cue conditions. Error bars represent \pm 1 SEM (within subjects). The asterisk indicates a significant difference between conditions $(* p<.05)$. RMSE $=$ root-mean-square error of the luminance values within the masked region of the face.

experiment in which we manipulated overall brightness of the faces in addition to local contrast (see Experiment S3 in the Supplemental Material). Participants judged faces with higher contrast around the eye region as more attractive even though the physical brightness of the faces was varied. Furthermore, participants judged the faces at the cued location as more attractive than the faces at the uncued location regardless of the overall brightness of the faces. These results are consistent with the interpretation that the attentional cue affected attractiveness judgments by enhancing perceived local contrast around the eyes and not by changing perceived overall brightness of the faces.

\section{General Discussion}

The question of whether attention alters observers' impressions of objects that they see has been a topic of debate for more than a century (Fechner, 1882; Helmholtz, 1866; James, 1890). Although studies showed that attention alters the appearance of simple stimuli (e.g., Carrasco et al., 2004), it was unclear whether attention also alters the appearance of real-world objects. We have shown that attention increases the perceived attractiveness of a face, demonstrating that attention can alter high-level aspects of perception. These findings illustrate the role of attention in forming initial subjective impressions of real-world stimuli, and faces in particular. In addition, they suggest that subjective aspects of perception-such as facial attractiveness — cannot be quantified solely by the intrinsic properties of a stimulus, but also depend, at least in part, on the observer's focus of exogenous attention.

In the present study, participants judged the face at the cued location to be more attractive than the face at the uncued location, but only when the faces were presented shortly after the cue. This finding is consistent with the known time course of exogenous attention (e.g., Carrasco et al., 2004; Nakayama \& Mackeben, 1989) and rules out the possibility that the observed cuing effects reflect decision or response biases. If participants were simply biased to pick the face at the cued location (and there were no changes in perceptual processing), this bias would be expected to affect responses across all conditions, regardless of the timing between the cue and face stimuli. Furthermore, the results of two control experiments in which participants were instructed to choose the face that appeared less attractive (Experiment S1) and 
the face that had lower contrast (Experiment S2) show that participants did not simply choose the stimulus at the cued location. Overall, these results are consistent with previous studies that used similar control procedures to rule out response biases as an explanation of results for tasks with simple stimuli (e.g., Carrasco et al., 2004; Fuller \& Carrasco, 2006; Gobell \& Carrasco, 2005; Montagna \& Carrasco, 2006). Furthermore, a recent electrophysiological study conclusively showed that effects of exogenous cues on perceived contrast in simple stimuli arise at an early processing stage in the visual pathways (Störmer et al., 2009; see also, Cutrone, Heeger, \& Carrasco, 2014; McDonald, Störmer, Martinez, Feng, \& Hillyard, 2013).

Although the present results point to fundamental consequences of attention in high-level perception in general, they also have important implications for research on facial attractiveness in particular. Studies on facial attractiveness have primarily focused on examining the intrinsic features of faces and have quantified how different features contribute to attractiveness judgments (for a review, see Rhodes, 2006). These studies have revealed a number of facial attributes that are consistently judged as attractive (e.g., symmetry and averageness; Langlois \& Roggman, 1990; Perrett et al., 1999). In the present study, the same face was perceived as more attractive when attention was drawn to it than when it was unattended. This indicates that perceived attractiveness of a face does not depend only on the face's physical attributes. Rather, perceived attractiveness of a face appears not to be fixed, even for a given observer, but to vary with the observer's focus of exogenous attention. Whether such effects on perceived facial attractiveness hold for other types of attention (e.g., voluntary attention), and the extent to which they persist with longer exposure of the faces, remains to be determined.

What is the mechanism underlying these attentioninduced changes in perceived attractiveness? Our data also show that participants judged faces with higher contrast around the eye region as more attractive than faces with lower contrast, which is consistent with previous work (Russell, 2003, 2009). We therefore propose that the attentional cue affected participants' attractiveness judgments by increasing apparent contrast at the location of the cue, that is, around the eye region of the faces; increased apparent contrast around the eyes, in turn, made the faces appear more attractive. Indeed, Experiment 3 showed that the attentional cue increased apparent local contrast around the eye region. This finding is in good agreement with previous studies showing that attention can increase the perceived contrast of simple stimuli (i.e., Gabor patches; e.g., Carrasco et al., 2004), but extends those results by showing that attention boosts perceived local contrast in real-world stimuli, such as faces. Furthermore, the present study shows the relevance of these attention-induced changes in appearance by demonstrating that they have direct influences on socially important judgments, that is, judgments of facial attractiveness. In future research, it will be important to investigate the ways in which attention affects attractiveness judgments in ecologically more valid situations, for example, during real-world interactions between people.

Overall, the present study shows that attention can alter aspects of high-level perception of real-world stimuli, such as faces. The findings indicate that in addition to altering the appearance of objects in a quantitative way (e.g., by making a face appear to have higher contrast), attention can modify subjective impressions of a face (e.g., by making it appear more attractive). They reveal that attention not only improves perceptual processing of stimuli encountered in the world, but also fundamentally changes the way that people experience these stimuli.

\section{Action Editor}

Philippe G. Schyns served as action editor for this article.

\section{Author Contributions}

V. S. Störmer performed the research and analyzed the data. V. S. Störmer and G. A. Alvarez designed the research and wrote the article.

\section{Acknowledgments}

We thank Molly Church and Stella Tu for helping with data collection and stimulus preparation.

\section{Declaration of Conflicting Interests}

The authors declared that they had no conflicts of interest with respect to their authorship or the publication of this article.

\section{Funding}

This work was supported by a Marie Curie fellowship (EU Grant PIOF-GA-2012-329920, to V. S. Störmer) and a National Science Foundation CAREER award (BCS-0953730, to G. A. Alvarez).

\section{Supplemental Material}

Additional supporting information can be found at http://pss sagepub.com/content/by/supplemental-data

\section{References}

Anton-Erxleben, K., Abrams, J., \& Carrasco, M. (2010). Evaluating comparative and equality judgments in contrast perception: Attention alters appearance. Journal of Vision, 10(11), Article 6. doi:10.1167/10.11.6

Anton-Erxleben, K., Henrich, C., \& Treue, S. (2007). Attention changes perceived size of moving visual patterns. Journal of Vision, 7(11), Article 5. doi:10.1167/7.11.5

Anton-Erxleben, K., Herrmann, K., \& Carrasco, M. (2013). Independent effects of adaptation and attention on perceived speed. Psychological Science, 24, 150-159. 
Brainard, D. H. (1997). The Psychophysics Toolbox. Spatial Vision, 10, 433-436.

Bronstad, P. M., \& Russell, R. (2007). Beauty is in the 'we' of the beholder: Greater agreement on facial attractiveness among close relations. Perception, 36, 1674-1681.

Carrasco, M., Fuller, S., \& Ling, S. (2008). Transient attention does increase perceived contrast of suprathreshold stimuli: A reply to Prinzmetal, Long, and Leonhardt (2008). Perception \& Psychophysics, 70, 1151-1164.

Carrasco, M., Ling, S., \& Read, S. (2004). Attention alters appearance. Nature Neuroscience, 7, 308-313.

Cunningham, M. R., Barbee, A. P., \& Pike, C. L. (1990). What do women want? Facialmetric assessment of multiple motives in the perception of male physical attractiveness. Journal of Personality and Social Psychology, 59, 61-72.

Cutrone, E. K., Heeger, D. J., \& Carrasco, M. (2014). Attention enhances contrast appearance via increased input baseline of neural responses. Journal of Vision, 14(14), Article 16. doi: $10.1167 / 14.14 .16$

Efran, M. G. (1974). The effect of physical appearance on the judgment of guilt, interpersonal attraction, and severity of recommended punishment in a simulated jury task. Journal of Research in Personality, 8, 45-54.

Fechner, G. T. (1882). Revision der hauptpunkte der psychophysik [Revision of the principles of psychophysics]. Leipzig, Germany: Breitkopf \& Härtel.

Fuller, S., \& Carrasco, M. (2006). Exogenous attention and color perception: Performance and appearance of saturation and hue. Vision Research, 46, 4032-4047.

Fuller, S., Rodriguez, R. Z., \& Carrasco, M. (2008). Apparent contrast differs across the vertical meridian: Visual and attentional factors. Journal of Vision, 8(1), Article 16. doi:10 $.1167 / 8.1 .16$

Gobell, J., \& Carrasco, M. (2005). Attention alters the appearance of spatial frequency and gap size. Psychological Science, 16, 644-651.

Helmholtz, H. von. (1866). Handbuch der physiologischen optik, Part III [Treatise on physiological optics]. Leipzig, Germany: Voss.

Hsieh, P. J., Caplovitz, G. P., \& Tse, P. U. (2005). Illusory rebound motion and the motion continuity heuristic. Vision Research, 45, 2972-2985.

James, W. (1890). The principles of psychology. New York, NY: Henry Holt.

Jonides, J. (1980). Voluntary versus automatic control over the mind's eye's movement. In J. B. Long \& A. Baddeley (Eds.), Attention and performance IX (pp. 187-203). Hillsdale, NJ: Erlbaum.

LaBerge, D. (1995). Attentional processing: The brain's art of mindfulness. Cambridge, MA: Harvard University Press.

Langlois, J. H., \& Roggman, L. A. (1990). Attractive faces are only average. Psychological Science, 1, 115-121.

Ling, S., \& Carrasco, M. (2007). Transient covert attention does alter appearance: A reply to Schneider (2006). Perception $\mathcal{E}$ Psychophysics, 69, 1051-1058.

Liu, T., Abrams, J., \& Carrasco, M. (2009). Voluntary attention enhances contrast appearance. Psychological Science, 20, 354-362.
Liu, T., Fuller, S., \& Carrasco, M. (2006). Attention alters the appearance of motion coherence. Psychonomic Bulletin $\mathcal{E}$ Review, 13, 1091-1096.

McDonald, J. J., Störmer, V. S., Martinez, A., Feng, W., \& Hillyard, S. A. (2013). Salient sounds activate human visual cortex automatically. The Journal of Neuroscience, 33, 9194-9201.

Montagna, B., \& Carrasco, M. (2006). Transient covert attention and the perceived rate of flicker. Journal of Vision, 6(9), Article 8. doi: $10.1167 / 6.9 .8$

Nakayama, K., \& Mackeben, M. (1989). Sustained and transient components of focal visual attention. Vision Research, 29, 1631-1647.

Pashler, H. E. (1998). The psychology of attention. Cambridge, MA: MIT Press.

Pelli, D. G. (1997). The VideoToolbox software for visual psychophysics: Transforming numbers into movies. Spatial Vision, 10, 437-442.

Perrett, D. I., Burt, D. M., Penton-Voak, I. S., Lee, K. J., Rowland, D. A., \& Edwards, R. (1999). Symmetry and human facial attractiveness. Evolution \& Human Behavior, 20, 295-307.

Perrett, D. I., Lee, K. J., Penton-Voak, I., Rowland, D., Yoshikawa, S., Burt, D. M., . . Akamatsu, S. (1998). Effects of sexual dimorphism on facial attractiveness. Nature, 394, 884-887.

Prinzmetal, W., Long, V., \& Leonhardt, J. (2008). Involuntary attention and brightness contrast. Perception \& Psychophysics, 70, 1139-1150.

Rhodes, G. (2006). The evolutionary psychology of facial beauty. Annual Review of Psychology, 57, 199-226.

Rhodes, G., Proffitt, F., Grady, J. M., \& Sumich, A. (1998). Facial symmetry and the perception of beauty. Psychonomic Bulletin E Review, 5, 659-669.

Rhodes, G., \& Tremewan, T. (1996). Averageness, exaggeration, and facial attractiveness. Psychological Science, 7, 105-110.

Russell, R. (2003). Sex, beauty, and the relative luminance of facial features. Perception, 32, 1093-1107.

Russell, R. (2009). A sex difference in facial contrast and its exaggeration by cosmetics. Perception, 38, 1211-1219.

Schneider, K. A. (2006). Does attention alter appearance? Perception \& Psychophysics, 68, 800-814.

Schneider, K. A., \& Komlos, M. (2008). Attention biases decisions but does not alter appearance. Journal of Vision, 8(15), Article 3. doi:10.1167/8.15.3

Störmer, V. S., McDonald, J. J., \& Hillyard, S. A. (2009). Crossmodal cueing of attention alters appearance and early cortical processing of visual stimuli. Proceedings of the National Academy of Sciences, USA, 106, 22456-22461.

Todorov, A., Mandisodza, A. N., Goren, A., \& Hall, C. C. (2005). Inferences of competence from faces predict election outcomes. Science, 308, 1623-1626.

Turatto, M., Vescovi, M., \& Valsecchi, M. (2007). Attention makes moving objects be perceived to move faster. Vision Research, 47, 166-178.

Willis, J., \& Todorov, A. (2006). First impressions: Making up your mind after a 100-ms exposure to a face. Psychological Science, 17, 592-598.

Wright, R. D., \& Ward, L. M. (2008). Orienting of attention. New York, NY: Oxford University Press. 